\title{
High-throughput genotyping for association mapping of agronomically important traits in rapeseed: a brief review of the current status
}

\author{
R.F. Gubaev ${ }^{1 *}$, S.V. Boldyrev ${ }^{1}$, D.V. Goryunov ${ }^{2}$, S.V. Goryunova ${ }^{3}$ \\ 1 Skolkovo Institute of Science and Technology, Moscow, Russia \\ ${ }^{2}$ Belozersky Institute of Physico-Chemical Biology, Moscow State University, Moscow Russia \\ ${ }^{3}$ Institute of General Genetics, Russian Academy of Science, Moscow, Russia
}

DOI 10.18699/ICG-PlantGen2019-75

(c) Autors, 2019

* e-mail: rimgubaev@gmail.com

\begin{abstract}
Rapeseed (Brassica napus) is one of the most important oilseed crops used for the production of vegetable oils. The key features that directly impact the quality and the quantity of the oil are agronomically important traits. The modern and effective approach that allows such traits to be improved is marker-assisted selection which relies on the socalled molecular markers, including DNA-based ones. In turn, to date, several approaches that facilitate the identification of a molecular marker, as well as genetic association mapping of phenotypical traits, have been implemented. Here we briefly review several recent studies devoted to association mapping of agronomically important traits in rapeseed by means of high-throughput genotyping technologies.

Key words: rapeseed; association studies; agronomically important traits; high throughput genotyping technologies.
\end{abstract}

\section{Introduction}

Rapeseed (Brassica napus) is an oilseed crop used worldwide for the production of vegetable oils. Rapeseed oil is the third most commonly produced vegetable oil in the world after palm and soy oil (List, 2016). In turn, the quality and the quantity of the oil are determined by phenotypic traits like plant architecture, oil composition, and resistance to unfavourable environmental conditions. Thus, the plant breeders try to improve such traits in order to increase the effectiveness of harvesting, quality and preservation of the yield. One of the ways to create lines with favourable properties is by marker-assisted selection. There are several types of molecular markers including microsatellites, single nucleotide polymorphisms (SNPs), restriction fragment length polymorphism. In the recent years, SNPs have become popular due to the rapid development of high-throughput genotyping techniques that are widely applied for association studies in rapeseed. These techniques could be conditionally separated into two types: those including high- and low-density DNA-based arrays and those based on high- throughput sequencing, include whole-genome sequencing (WGS), genotyping by sequencing (GBS), and associative transcriptomics (Perez-de-Castro et al., 2012). In the present review, several studies that implement high-throughput genotyping for association mapping of agronomically important traits in rapeseed are discussed.

\section{Plant architecture}

Traits related to plant architecture in rapeseed include plant height, primary branch number, branching angle. Plant architecture affects the effectiveness of photosynthesis, harvest convenience, and so several attempts have been made in order to determine underlying genetic factors. The SNPs associated with plant height were shown to be located close to the genes that encode enzymes related to the metabolism of gibberellins, which, in turn, regulate growth processes in plants $(\mathrm{Li}$ et al., 2016a). The novel markers for primary branch number were found in chromosome $\mathrm{C}$, all of them were adjacent to genes that encode transcription factors involved in plant development control (Li et al., 2016a). In the recent studies, Liu et al. (2016) identified potential markers associated with branch angle, the SNPs were located close to genes that encode enzymes for the metabolism of auxin which, in turn, regulates cell elongation. Another study by Li et al. (2017) also revealed several markers for the branch angle. It was demonstrated that potential candidate genes were presented by LAZY1 and TAC1 from the IGT gene family, which had been previously shown to control branch angle, leaf angle and shoot gravitropism in crops.

\section{Oil composition}

There are several compounds that are usually considered as ones affecting the quality of rapeseed's vegetable oil, namely fatty acid (oleic, linoleic, erucic acids), glucosinolates, tocopherols. Glucosinolates, the secondary metabolites produced by Brassicaceae plants, and erucic acid are the compounds that reduce the quality of the oil by making it toxic and inappropriate for baking. In contrast, unsaturated fatty acids, namely oleic and linoleic as well as tocopherols possessing vitamin $\mathrm{E}$ activity, are considered as beneficial components of the oil. Several studies indicate that glucosinolate content is related to SNP adjacent genes that encode glucosinolate transporters and various transcription factors (Qu et al., 2015, Li et al., 2014, Wei et al., 2019). Loci associated with erucic acid content were present on chromosomes A8, A9, A10, C3, C8, C9 (Li et al., 2014; Havlickova et al., 2017; Qu et al., 2017). The markers associated with fatty acid content were shown to be located close to the genes encoding fatty acid elongases, 3-ketoacyl-CoA synthases, and fatty acid desaturases (Gacek et al., 2017; Qu et al., 2017). Using the associative transcriptomics approach, the ortholog of the VTE gene, 
which encodes tocopherol methyl transferase, was shown to determine tocopherol compositions (Havlickova et al., 2017).

\section{Phenology}

Phenological traits that affect the potential of rapeseed yield include flowering time, maturity time, earliness; these traits generally determine how much time plants invest in vegetative growth as well as in seed formation. The trait 'flowering time' has been widely studied in rapeseeds. Thus, potential flowering time markers were found near the following genes: CONSTANS, PHYTOCHROME B, FRIGIDA, FLOWERING LOCUS T, FRUITFUL, FLOWERING LOCUS C, which has been previously demonstrated to regulate photoperiodic reactions in thale cress plants (Xu et al., 2015; Raman et al., 2016). Phenological traits were more deeply investigated by Zhou et al. (2017) who were searching for associations for the timing of initial flowering, maturity and final flowering as well as the flowering period. It was demonstrated that these traits are related to 131 SNPs located within the loci that contain genes responsible for the flowering process.

\section{Resistance to unfavourable environmental conditions}

One of the key features that determine the yield amount is the ability to resist to biotic as well as abiotic environmental factors, which include high salinity, freezes, pathogens. Several recent association studies revealed genes and potential markers responsible for resistance to pathogens and unfavourable abiotic factors. Markers significantly associated with the resistance of rapeseed to Sclerotinia stem rot were found adjacent to the genes related to lignin biosynthesis, jasmonic acid pathway, defence response (Wei et al., 2016). Another study revealed associations with clubroot disease, notably, all potential markers were shown to be located near the genes that encode disease resistance proteins of the TIR-NBS family (Li et al., 2016b). Salt resistance markers were found by Wan et al. (2017), all SNPs were adjacent to salt-stress related genes encoding aquaporins, enzymes involved in the biosynthesis of proline. Electrolyte leakage was assessed in order to reveal markers for the freezing tolerance of rapeseed, several associated loci were shown to contain genes that encode sulfur transport proteins, enzymes involved in abscisic acid biosynthesis, transcription factors related to development (Fiebelkorn et al., 2018).

\section{Conclusions}

Since rapeseed is an agronomically important plant for vegetable oil production, the number of studies on genetic factors underlying phenotype traits is constantly increasing. The most recent studies are generally devoted to traits related to plant architecture, oil composition, phenology and resistance to unfavourable environmental conditions. It should also be mentioned that modern high-throughput genotyping technologies have become more popular for these purposes due to high performance and relatively low costs. Recent insights in phenotype-genotype associations in rapeseed will facilitate the uncovering of new markers for agronomically important traits, which, in turn, will facilitate the improvement of rapeseed lines as well as the creation of new ones.

\section{References}

Fiebelkorn D., Horvath D., Rahman M. Genome-wide association study for electrolyte leakage in rapeseed/canola (Brassica napus L.). Mol. Breeding. 2018;38(11):129.

Gacek K., Bayer P.E., Bartkowiak-Broda I., Szala L., Bocianowski J., Edwards D., \& Batley, J. Genome-wide association study of genetic control of seed fatty acid biosynthesis in Brassica napus. Front. Plant Sci. 2017;7:2062.

Havlickova L., He Z., Wang L., Langer S., Harper A.L., Kaur H., Bancroft I. Validation of an updated Associative Transcriptomics platform for the polyploid crop species Brassica napus by dissection of the genetic architecture of erucic acid and tocopherol isoform variation in seeds. Plant Journal. 2018;93(1):181-192.

Li F., Chen B., Xu K., Gao G., Yan G., Qiao J., Zhang T. A genomewide association study of plant height and primary branch number in rapeseed (Brassica napus). Plant Sci. 2016a;242:169-177.

Li F., Chen B., Xu K., Wu J., Song W., Bancroft I., Wang N. Genomewide association study dissects the genetic architecture of seed weight and seed quality in rapeseed (Brassica napus L.). DNA Research. 2014;21(4):355-367.

Li H., Zhang L., Hu J., Zhang F., Chen B., Xu K., Wu X. Genome-wide association mapping reveals the genetic control underlying branch angle in rapeseed (Brassica napus L.). Front. Plant Sci. 2017;8:1054.

Li L., Luo Y., Chen B., Xu K., Zhang F., Li H., Li F. A genome-wide association study reveals new loci for resistance to clubroot disease in Brassica napus. Front. Plant Sci. 2016b;7:1483.

List G.R. Oilseed Composition and Modification for Health and Nutrition. Functional Dietary Lipids. Woodhead Publishing. 2016

Liu J., Wang W., Mei D., Wang H., Fu L., Liu D., Hu Q. Characterizing variation of branch angle and genome-wide association mapping in rapeseed (Brassica napus L.). Front. Plant Sci. 2016a;7:21.

Perez-de-Castro A., Vilanova S., Cañizares J., Pascual L., Blanca J., Diez M., Picó B. Application of genomic tools in plant breeding. Current Genomics. 2012;13(3):179-195.

Qu C., Jia L., Fu F., Zhao H., Lu K., Wei L., Li J. Genome-wide association mapping and Identification of candidate genes for fatty acid composition in Brassica napus L. using SNP markers. BMC Genomics. 2017;18(1):232.

Qu C.M., Li S.M., Duan X.J., Fan J.H., Jia L.D., Zhao H.Y., Wang R. Identification of candidate genes for seed glucosinolate content using association mapping in Brassica napus L. Genes. 2015;6(4): 1215-1229.

Raman H., Raman R., Coombes N., Song J., Prangnell R., Bandaranayak C., Dennis E.S. Genome-wide association analyses reveal complex genetic architecture underlying natural variation for flowering time in canola. Plant, Cell Environment. 2016;39(6):1228-1239.

Wan H., Chen L., Guo J., Li Q., Wen J., Yi B., Shen J. Genome-wide association study reveals the genetic architecture underlying salt tolerance-related traits in rapeseed (Brassica napus L.). Front. Plant Sci. 2017;8:593.

Wei D., Cui Y., Mei J., Qian L., Lu K., Wang Z.M., Qian W. Genomewide identification of loci affecting seed glucosinolate contents in Brassica napus L. J. Integrative Plant Biol. 2019;61(5):611-623.

Wei L., Jian H., Lu K., Filardo F., Yin N., Liu L., Li J. Genome-wide association analysis and differential expression analysis of resistance to Sclerotinia stem rot in Brassica napus. Plant Biotechnol. J. 2016;14(6):1368-1380.

Xu L., Hu K., Zhang Z., Guan C., Chen S., Hua W., Ma C. Genome-wide association study reveals the genetic architecture of flowering time in rapeseed (Brassica napus L.). DNA Research. 2015;23(1):43-52.

Zhou Q., Han D., Mason A.S., Zhou C., Zheng W., Li Y., Huang Y. Earliness traits in rapeseed (Brassica napus): SNP loci and candidate genes identified by genome-wide association analysis. $D N A$ Research. 2017;25(3):229-244.

Acknowledgements. This study was supported by the Ministry of Science and Higher Education of the Russian Federation (Grant No. 14.609.21.0099).

Conflict of interest. The authors declare no conflict of interest. 